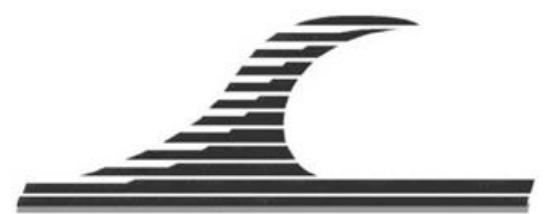

Revue Paralia, Volume 7 (2014) pp s04.1-s04.12

Mots-clés : Structure poreuse, Surface spécifique, Perte de charge, Houle, Réflexion, Dissipation.

(C) Editions Paralia CFL

\title{
Ecoulements stationnaires et oscillants à travers des milieux poreux : effets de la surface spécifique
}

\author{
Gwendoline ARNAUD ${ }^{1,2}$, Julien TOUBOUL ${ }^{1}$, Damien SOUS ${ }^{1}$, \\ Fabrice GOUAUD ${ }^{2}$, Vincent REY ${ }^{1}$
}

1. Université de Toulon, Aix-Marseille Université, CNRS/INSU, IRD, MIO, UM 110, 83957, La Garde Cedex, France. garnaud@univ-tln.fr,sous@univ-tln.fr,touboul@univ-tln.fr, rey@univ-tln.fr

2. ACRI-In, 260, route du Pin Montard Sophia-Antipolis F-06410 Biot, France.

Fabrice.Gouaud@acri-in.fr

\section{Résumé :}

Le but de ce travail était l'étude des effets de la surface spécifique sur l'énergie dissipée à travers un milieu poreux "modèle" constitué d'un réseau de cylindres verticaux émergeants. Les expériences ont été effectuées dans un canal hydraulique long de dix mètres. Trois milieux poreux ont été utilisés avec des cylindres de diamètres différents. Les modèles de structures poreuses étaient constitués par des réseaux réguliers de cylindres de diamètres constants. L'effet de la surface spécifique a été analysé en utilisant trois diamètres de cylindres différents tout en maintenant la porosité constante. Deux séries d'expériences sont présentées. D’une part, les mesures dans des conditions d'écoulement stationnaire, avec des vitesses et des profondeurs différentes, ont démontré l'influence significative de la surface spécifique sur la perte de charge à travers les milieux poreux. D’autre part, une seconde série d'expériences a mis l'accent sur la propagation de houles régulières à travers les structures poreuses, montrant le rôle joué par la surface spécifique à la fois sur l'atténuation des vagues et sur les processus d'interférences. L'amortissement observé était d'autant plus grand que la surface spécifique est grande.

Article issu d'une sélection des XIIIèmes Journées Nationales Génie Côtier Génie Civil qui se sont tenues à Dunkerque du 2 au 4 juillet 2014.

Soumis le 28 novembre 2014, accepté le 9 décembre 2014, en ligne le 22 décembre 2014.

La seule version examinée est celle écrite en français. La ou les autres versions n'étant pas examinées par le comité de rédaction de la revue, sont donc publiées sous l'entière responsabilité du ou des auteurs.

A TRANSLATED VERSION IN ENGLISH IS AVAILABLE ONLINE

Pour citer cet article :

ARNAUD G., TOUBOUL J., SOUS D., GOUAUD F., REY V. (2014). Ecoulements stationnaires et oscillants à travers des milieux poreux : effets de la surface spécifique. Revue Paralia, Vol. 7, pp s04.1s04.12.

DOI: http://dx.doi.org/10.5150/revue-paralia.2014.s04 


\section{Introduction}

Les zones côtières sont particulièrement exposées, et vulnérables, à l'énergie incidente de la houle. Des structures ont été conçues depuis des décennies pour atténuer l'effet des vagues sur le littoral ou sur les aménagements côtiers. Parmi les premières approches scientifiques, CALHOUN (1971) a étudié la digue à talus en enrochement pour le port de Monterey (Californie). Ces mesures pionnières sur site ont montré le rôle joué par ces structures côtières perméables sur la dynamique des vagues, induisant une réflexion et une transmission de la houle jusqu'à $40 \%$ et 10 à $20 \%$, respectivement. La dissipation d'énergie dans la structure est causée par des processus d'interférences et de réflexions multiples. Quand les vagues se propagent au-dessus du milieu poreux, l'atténuation est souvent liée aux effets inertiels et non-linéaires (GU \& WANG, 1991, et références citées) qui peuvent être pris en compte en utilisant une relation de dispersion complexe. SOLLITT \& CROSS (1972) ont étudié expérimentalement la transmission et la réflexion de la houle par des brise-lames perméable de différentes formes. Le coefficient de réflexion a été observé plus faible avec une largeur de digue et une longueur d'onde croissantes, ainsi qu'une porosité et une perméabilité décroissantes. Inversement, le coefficient de transmission diminue avec une longueur d'onde, une porosité et une perméabilité décroissantes, ainsi qu'une hauteur d'eau et une largeur de digue croissantes. MADSEN (1983) a proposé une solution théorique de la réflexion de la houle linéaire par un mur poreux dans le cas eau peu profonde. Il a exprimé le coefficient de réflexion en fonction des paramètres de la houle incidente et des propriétés de la structure poreuse (largeur de la structure, diamètre des grains et porosité). L'analyse de la transmission et de la réflexion de la houle par une structure perméable a été étendue au cas de la houle incidente oblique sur un mur poreux par DALRYMPLE (1991). La dynamique des vagues à travers des blocs poreux superposés a été étudiée par YU \& CHWANG (1994) pour des conditions de dissipation faibles à fortes, incluant l'influence des modes évanescents lié aux discontinuités d’indices des milieux dans la direction de propagation. Bien que chacun de ces modèles soit fondé sur une représentation linéaire des effets de dissipation, les écoulements à grand nombre de Reynolds peuvent être décrits par une approche quadratique (MOLIN, 2011) appliqué pour des milieux poreux minces.

La première section de ce papier présente le dispositif et les méthodes expérimentales. La seconde partie est dédiée à la description des résultats expérimentaux avec, d'une part, l'étude de la perte de charge d'un écoulement permanent à travers des milieux poreux et, d'autre part, l'analyse de la dynamique des vagues à travers des milieux poreux incluant une étude en fréquence des coefficients de réflexion et de transmission. Une attention particulière est portée sur le rôle joué par la surface spécifique définie comme la surface fluide-contact par unité de volume poreux (GUYON et al., 1991). Conclusions et perspectives sont présentées dans la dernière section de cet article. 
Ecoulements stationnaires et oscillants à travers des milieux poreux : effets de la surface spécifique : s04.3

\section{Dispositif expérimental}

\subsection{Canal hydrodynamique et milieux poreux}

Les expériences ont été réalisées dans le canal à houle de SeaTech à Toulon, France. Ce canal houle/courant est long de $10 \mathrm{~m}$, large de $0.3 \mathrm{~m}$ et haut de $0.5 \mathrm{~m}$.

Le milieu poreux modèle est constitué d'un réseau dense de cylindres verticaux émergeants, uniformément disposés le long de deux axes perpendiculaires formant un angle de $45^{\circ}$ avec l'axe longitudinal (figure 1). Trois diamètres de cylindres, $D=0.020,0.032$ et $0.050 \mathrm{~m}$, ont été utilisés. La porosité est constante et égale à 0.7. La surface spécifique $s$ diminue lorsque $D$ augmente et vaut respectivement 52 , 33, et $22 \mathrm{~m}^{-1}$ pour les diamètres mentionnés ci-dessus. La longueur de la structure poreuse varie pendant les expériences (voir ci-après).
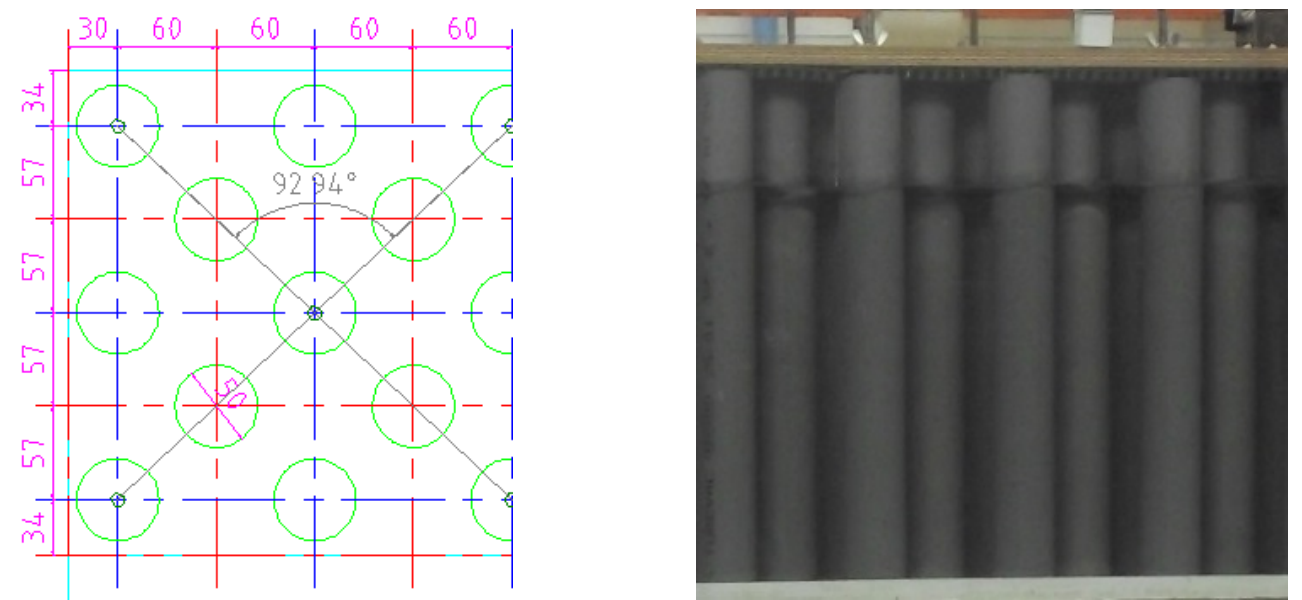

Figure 1. Schémas de la vue du dessus (gauche) et photo de la vue de côté (droite) de la géométrie des cylindres dans le milieu poreux

\subsection{Conditions expérimentales et instrumentation}

Le niveau de la surface libre est mesuré grâce à 7 sondes à houle résistives synchrones, avec une fréquence d'échantillonage de $32 \mathrm{~Hz}$. Des mesures de vitesses à haute fréquence $(200 \mathrm{~Hz})$ ont été effectuées grâce à 5 courantomètres à effet Doppler (Vectrino ${ }^{\odot}$ de NortekMed).

Dans le cas d'un écoulement stationnaire, la longueur de la structure poreuse était $L=4.80 \mathrm{~m}$. Les cinq courantomètres synchronisés étaient installés respectivement en amont, tous les $1.20 \mathrm{~m}$ dans le milieu poreux et en aval du poreux. Les gammes des débits et des vitesses étudiés varient entre $3 \times 10^{-3}$ et $16 \times 10^{-3} \mathrm{~m}^{3} / \mathrm{s}$ et 0.04 et $0.20 \mathrm{~m} / \mathrm{s}$, respectivement (noter que l'élévation de surface libre n’est pas maintenue constante). 


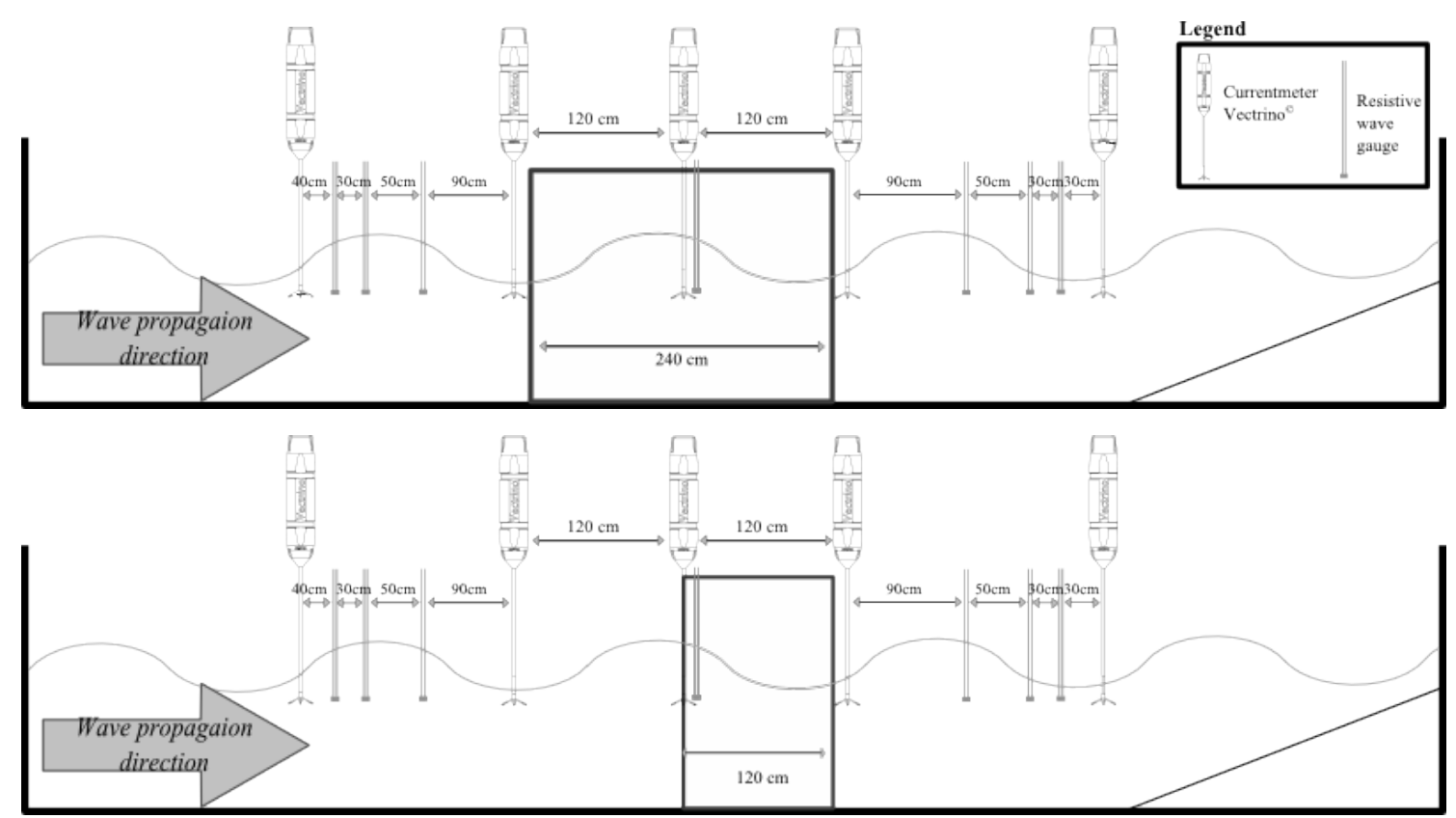

Figure 2. Schémas du dispositif expérimental pour l'écoulement oscillant, haut :

$$
L=2.40 \mathrm{~m} \text { et bas : } L=1.20 \mathrm{~m} \text {. }
$$

Les expériences de houle ont été menées pour deux longueurs $L$ de poreux, respectivement 1.20 et $2.40 \mathrm{~m}$, pour une hauteur d'eau constante $h=0.23 \mathrm{~m}$. La gamme de périodes étudiées est 0.55-2.00 s. Les positions des sondes à houle (voir figure 2) sont choisies afin de caractériser la réflexion, la transmission et l'atténuation de la houle à l'aide de la méthode à trois sondes (MANSARD \& FUNKE, 1980). En outre, cinq courantomètres ont été positionnés de chaque côté et à l'intérieur de la structure. Une plage en pente douce est située à l'extrémité aval du canal afin de maximiser la dissipation de l'énergie des vagues transmises et d’éviter des réflexions parasites.

\section{Résultats expérimentaux}

\subsection{Perte de charge pour un écoulement stationnaire}

Les effets inertiels sont généralement négligés pour des écoulements à faible nombre de Reynolds. La loi de Darcy est alors utilisée pour définir le débit $Q$ à travers une surface $S$ comme une fonction du gradient de pression $\triangle P$ :

$Q=U . S=-\frac{K}{\mu} \frac{\Delta P}{L}$

Où $\mu$ est la viscosité du fluide, $U$ la vitesse moyenne de l'écoulement et $K$ la perméabilité intrinsèque du milieu poreux, qui est une fonction de la géométrie du milieu, et $L$ est la longueur du milieu poreux. 
Pour les régimes d'écoulement plus élevés, les effets inertiels doivent être pris en compte en ajoutant un terme de type Forchheimer, conduisant à l'expression quadratique suivante :

$\frac{\Delta P}{L}=\alpha U+\beta U^{2}$

L’équation 2 peut être réécrite sous la même forme que la loi de Darcy (voir éq. 3) dans laquelle la perméabilité dépend de la vitesse de l'écoulement comme $K_{a p p}=K(U)$ (voir éq. 4).

$U . S=-\frac{K}{\mu(\alpha+\beta U)} \frac{\Delta P}{L}$

$K_{\text {app }}=\frac{K}{\alpha+\beta U}$

La figure 3 représente la perte de charge en fonction de la vitesse en amont. On note tout d'abord que le gradient de pression n'est pas une fonction linéaire de la vitesse, indiquant des effets inertiels non-négligeables. Le gradient de pression tend à augmenter, comme prévu, avec la vitesse de l'écoulement, mais aussi à diminuer avec l'augmentation du diamètre des cylindres. Ceci démontre l'effet de la surface spécifique sur la perte de charge à porosité constante.

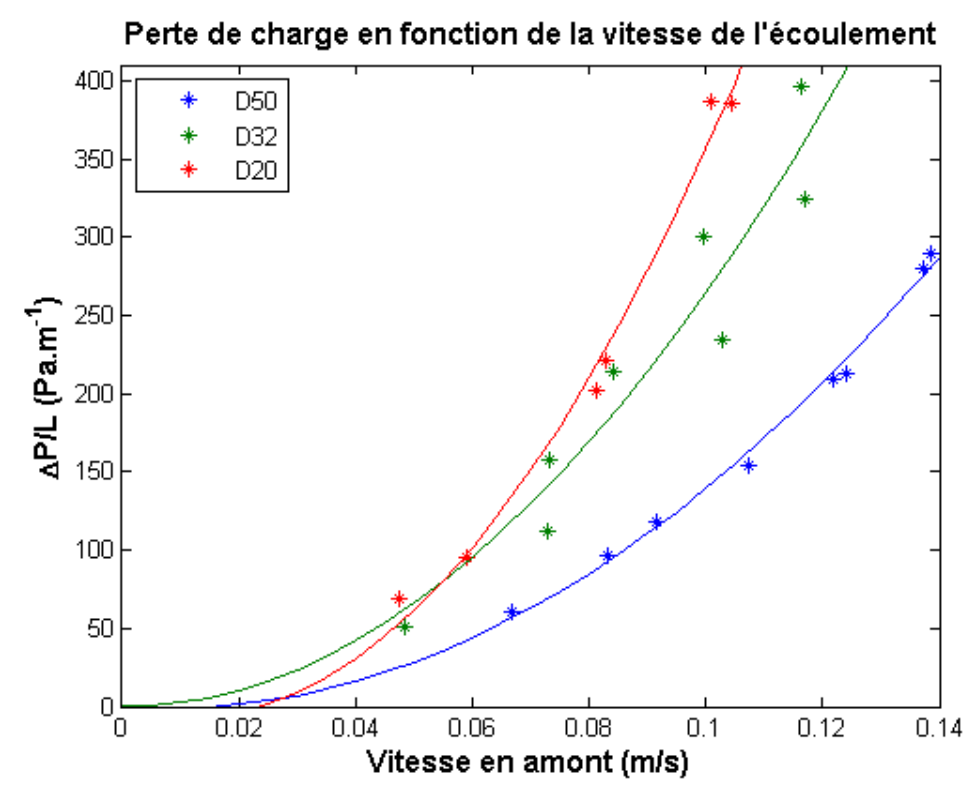

Figure 3. Perte de charge en fonction de la vitesse amont pour les trois diamètres de cylindres, écoulement stationnaire.

Dans le but de comparer les résultats expérimentaux à la théorie, le taux de l'amortissement est calculé comme suit : 
$\varepsilon_{D}=\int_{h} F_{d} N_{t} U d h=N_{t} \int_{h} F_{d} U d h$

où $\varepsilon_{D}$ est le taux d'énergie dissipée calculé sur la base de l'hypothèse de cylindres distincts, $F_{d}$ est la contrainte de trainée, $N_{t}$ est le nombre de cylindres dans la structure poreuse, $U$ est la vitesse moyenne et $h$ est la profondeur d'eau. Ce terme de puissance dissipée correspond à l'intégrale sur la profondeur de la force locale exercée par la contrainte de trainée sur les cylindres. La contrainte de trainée $F_{d}=1 / 2 \times \rho U^{2} A C_{d}$ est définie sur un cylindre de section transversale $A$ pour un fluide de densité $\rho$.

Le coefficient $C_{d}$ est ajusté empiriquement pour chaque poreux afin de correspondre aux mesures. La valeur 1.2 est suggérée pour un unique cylindre lisse (MUNSON et al., 1990). L’énergie dissipée calculée expérimentalement est proportionnelle à la perte de charge à travers la structure poreuse :

$\varepsilon_{D}=\rho g h_{L}$

Où $h_{L}=\frac{\left(h_{2}-h_{1}\right)^{3}}{4 \mathrm{~h}_{1} h_{2}}$ est la perte de charge calculée à l'aide de l'élévation de surface libre amont et aval.

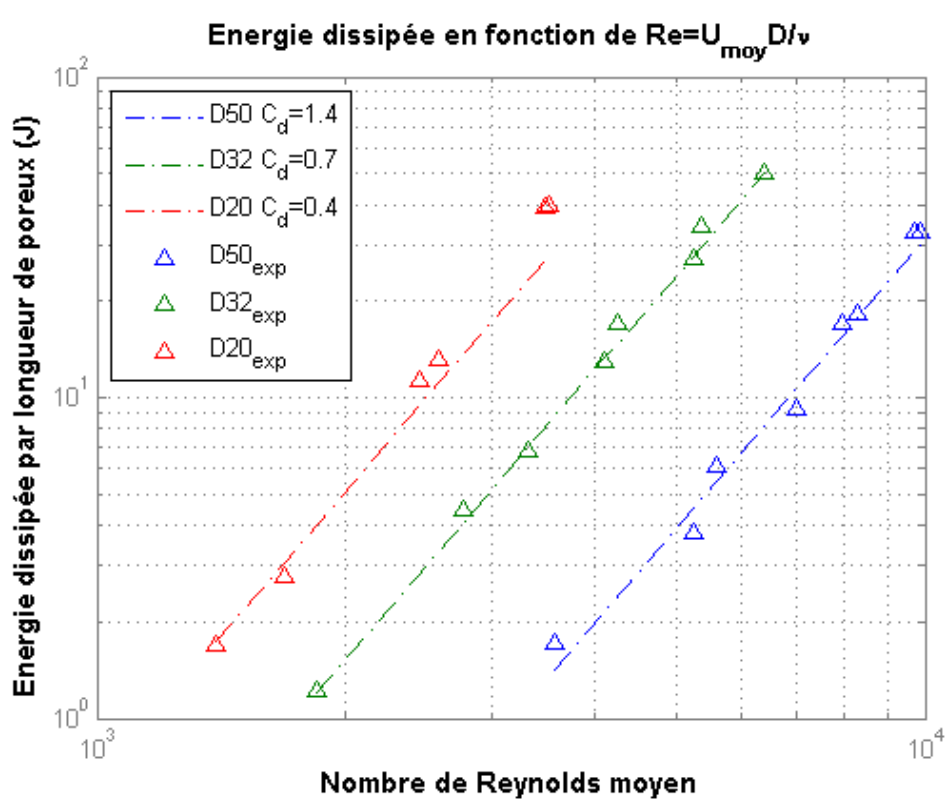

Figure 4. Energie dissipée en fonction du nombre de Reynolds calculé en amont de la structure poreuse $R e=\left(U_{\text {moy }} D\right) / v$, avec $D$ le diamètre des cylindres.

La figure 4 présente les valeurs de l'énergie dissipée issues des expériences, calculées à partir de l'équation 6 avec le meilleur ajustement de l'expression théorique (éq. 5). Le coefficient de trainée est un paramètre ajusté empiriquement. On note, tout d'abord le bon accord entre les mesures et la théorie ajustée. L'énergie dissipée, pour un diamètre donné, augmente avec le nombre de Reynolds et, inversement, augmente avec le 
diamètre décroissant pour un nombre de Reynolds donné. Cela souligne à nouveau le rôle joué par la surface spécifique sur la dynamique de l'écoulement. Les pentes des droites sont très semblables, indiquant une dépendance du nombre de Reynolds et une tendance à être indépendante du diamètre. Les coefficients de trainée ajustés augmentent avec le diamètre, avec $C_{d}=0.4,0.7$ et 1.4 respectivement pour $D=0.020,0.032$ et $0.050 \mathrm{~m}$.

\subsection{Réflexion, transmission et atténuation des vagues}

\subsubsection{Approche théorique}

La théorie proposée ici pour décrire le comportement de la houle à travers un milieu poreux est basée sur une approche linéaire, c'est-à-dire que les hypothèses considérées sont une faible amplitude de l'onde et un écoulement du fluide qui dérive d'un potentiel des vitesses. D’autres hypothèses typiques sont une échelle caractéristique de l'atténuation de la houle beaucoup plus grande que la longueur d'onde et une amplitude des vagues qui décroît de façon exponentielle ce qui correspond à un taux de dissipation constant le long de l'axe de propagation. La longueur du canal est divisée en trois parties $(j=1,2,3)$ respectivement amont, milieu poreux et aval. L'origine des abscisses correspond au début du milieu poreux. Une méthode intégrale est utilisée pour résoudre ce problème (REY et al., 1992 ; REY, 1995). Le potentiel des vitesses est calculé pour chaque domaine en utilisant l'expression suivante :

$\Phi_{j}(x, z, t)=\varphi_{j}(x, z) e^{i(\omega t)}=A_{j}^{ \pm} e^{ \pm\left(i k_{j} X\right)} \cosh \left(k_{j, p}(z+h)\right) e^{i(\omega t)}$

Avec $k_{j}=k_{j, p}-i k_{j, d}$ le nombre d'onde, complexe dans le milieu poreux avec un terme dissipatif, et $\omega=2 \pi f=2 \pi / T$ la fréquence angulaire de la houle. Les inconnues sont les coefficients $A_{j}^{ \pm}$. Le terme dissipatif $k_{j, d}$ est supposé nul de chaque côté de la structure et est choisi de la forme $k_{j, d}=k_{j, p} / n$ dans le milieu poreux, où $n$ correspond à un taux d'atténuation par unité de longueur d'onde indépendant de la fréquence. Les modes évanescents, significatifs en présence d'une discontinuité de la bathymétrie, ou de la nature du milieu dans la colonne d’eau, sont négligés ici.

Considérant les conditions aux frontières, la continuité des vitesses et des pressions aux interfaces $x=0$ et $x=L$ (YU \& CHWANG, 1994) s'écrivent :

$\varphi_{i}=S_{r} \varphi_{j} \quad$ et $\quad \frac{\partial \varphi_{i}}{\partial x}=\gamma \frac{\partial \varphi_{j}}{\partial x}$

Où $i$ est l'indice du domaine en amont $(x \leq 0)$ et en aval de la structure poreuse $(x \geq L)$ et $j$ est l'indice du poreux.

$S_{r}$ est la réactance du milieu définie par l'équation 9, avec $C_{m}$ le coefficient de masse ajoutée. Ce coefficient est nul en amont et en aval du poreux. Dans le milieu poreux, $C_{m}$ est utilisé comme un paramètre ajustable pour adapter le modèle théorique aux expériences. 


$$
S_{r}=1+C_{m} \frac{1-\gamma}{\gamma}
$$

Finalement, la forme générale de la relation de dispersion (YU \& CHWANG, 1994), en ne gardant que les effets inertiels, est :

$$
\omega^{2} S_{r}=g k_{j, p} \tanh \left(k_{j, p} h\right)
$$

La longueur d'onde dans le poreux est d'autant plus faible que $S_{r}$ est importante. Elle est ici uniquement fonction du coefficient de masse ajoutée $C_{m}$ pour une porosité constante $\gamma$.

\subsubsection{Résultats : réflexion et atténuation d'une houle régulière}

La réflexion de la houle par les structures poreuses et par la plage dissipative en aval ainsi que la transmission à travers les milieux poreux sont mesurées pour quantifier les effets du réseau de cylindres. Les figures 5 à 10 montrent l'évolution des coefficients de réflexion et de transmission par une étude en fréquence pour chacune des expériences. Les traits pleins et les croix représentent les prédictions du modèle théorique et les résultats expérimentaux, respectivement.

Les résultats expérimentaux, montrent que, pour une longueur donnée de la structure poreuse, la transmission diminue avec l'augmentation du diamètre des cylindres et une fréquence croissante. Cette dernière tendance n'est cependant observée qu'à partir d'une fréquence supérieure à $1 \mathrm{~Hz}$. Pour des fréquences inférieures, la transmission semble être constante. Cette observation est discutée plus tard dans la comparaison entre la théorie et les expériences.

En ce qui concerne la réflexion, on observe tout d'abord que la réflexion sur la plage dissipative est presque négligeable dans chacun des cas examinés. La réflexion par la structure poreuse présente un caractère oscillant par rapport à la fréquence. Ces oscillations sont liées aux processus d'interférences dues à deux sauts successifs d'indice du milieu aux frontières $x=0$ et $x=L$. Ces oscillations sont plus nombreuses pour les plus grands diamètres et pour les structures poreuses plus courtes, c'est-à-dire pour une faible dissipation et une transmission plus élevée. A haute fréquence, les oscillations du coefficient de réflexion liées au phénomène d’interférences diminuent et tendent vers une valeur constante, d'environ 0.2 , ce qui correspond à un milieu poreux de longueur infini.

Le modèle a été calibré avec les paramètres mentionnés ci-dessus en utilisant les données expérimentales à hautes fréquences où la décroissance du coefficient de transmission avec l'augmentation de la fréquence est bien représentée. Les deux paramètres ajustables $C_{m}$ et $n$ jouent un rôle différent dans la modélisation de la dynamique de la houle. La masse ajoutée $C_{m}$ n'affecte pas l'atténuation mais contrôle la longueur d'onde dans le milieu poreux. Ce paramètre est donc ajusté afin d'optimiser la description des modes d'oscillation du coefficient de réflexion dans le domaine 
Ecoulements stationnaires et oscillants à travers des milieux poreux : effets de la surface spécifique : s04.9

fréquentiel. A ce stade de l'étude, $C_{m}$ est supposé constant et indépendant du diamètre des cylindres et de la longueur de la structure poreuse. Le coefficient d'atténuation $n$ agit principalement sur la transmission à travers la structure poreuse via le terme d'amortissement $k_{2, d}=k_{2, p} / n$ et, en tant que tel, a été ajusté pour représenter au mieux la décroissance du coefficient de transmission pour les plus hautes fréquences (voir cidessus pour l'écart entre le modèle et les expériences pour la gamme de fréquence où la transmission constante). Les prédictions théoriques les plus ajustées sont finalement obtenus avec $C_{m}=0.3$ et $n=13,20$ et 25 pour $D=0.020,0.032$ et $0.050 \mathrm{~m}$, respectivement.

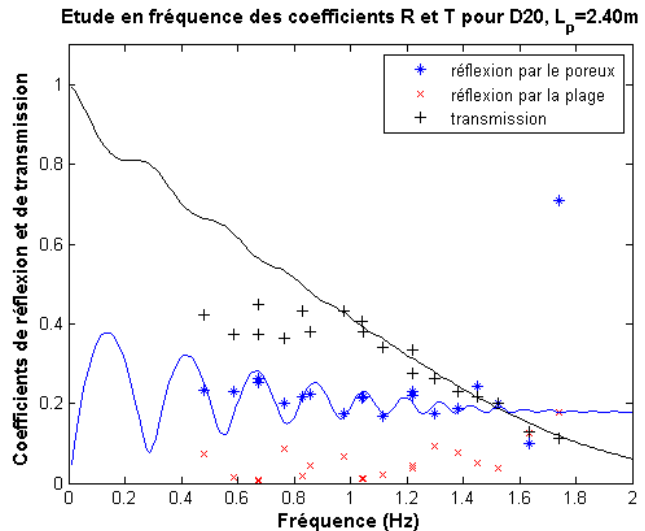

Figure 5. Coefficients de réflexion et de transmission, $L=2.40 \mathrm{~m}, D=0.020 \mathrm{~m}$

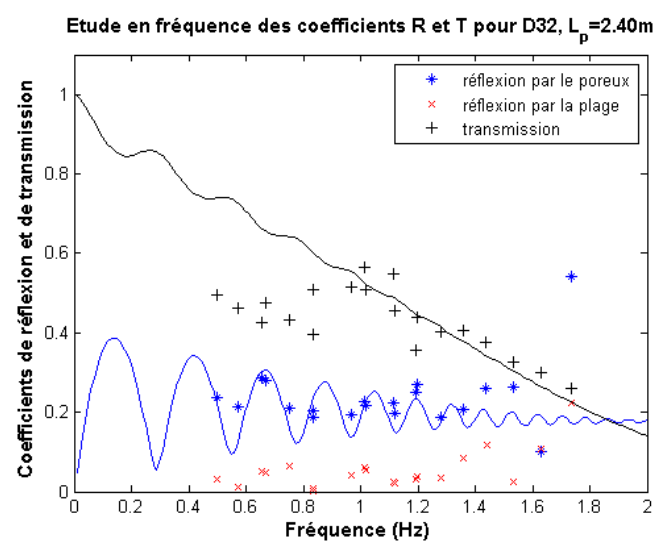

Figure 7. Coefficients de réflexion et de transmission $L=2.40 \mathrm{~m}, D=0.032 \mathrm{~m}$.

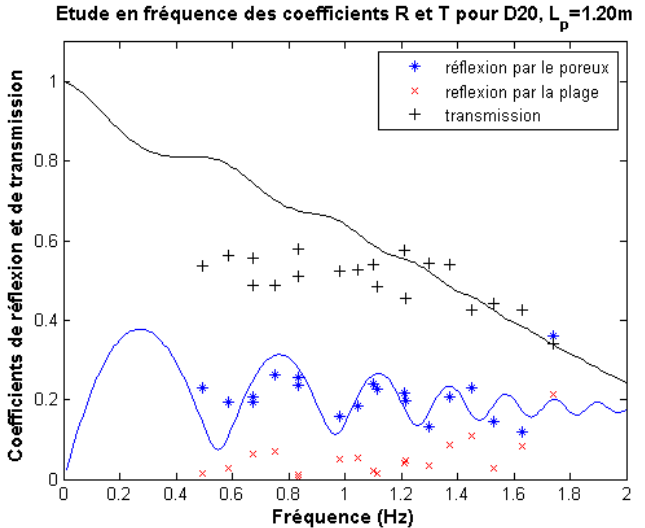

Figure 6. Coefficients de réflexion et de transmission, $L=1.20 \mathrm{~m}, D=0.020 \mathrm{~m}$

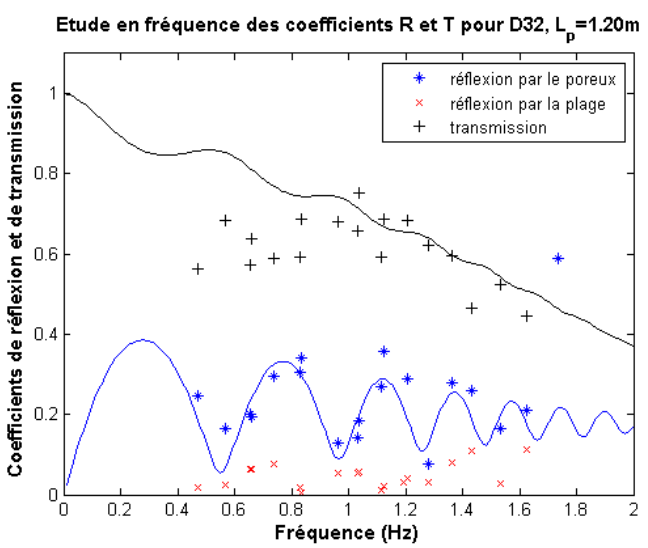

Figure 8. Coefficients de réflexion et de transmission, $L=1.20 \mathrm{~m}, D=0.032 \mathrm{~m}$. 


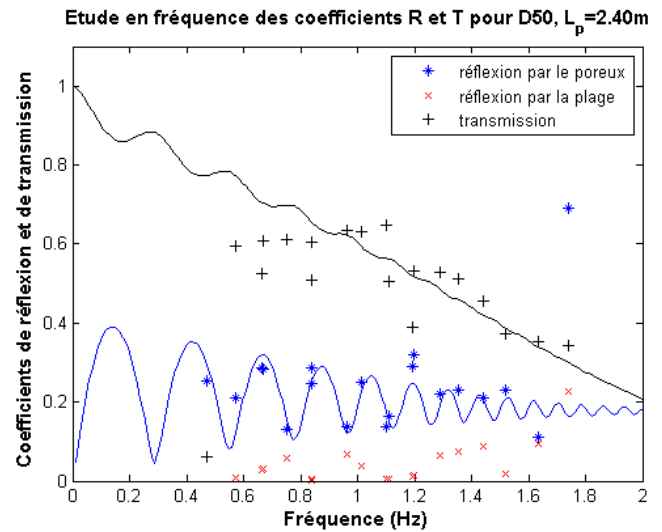

Figure 9. Coefficients de réflexion et de transmission, $L=2.40 \mathrm{~m}, D=0.050 \mathrm{~m}$.

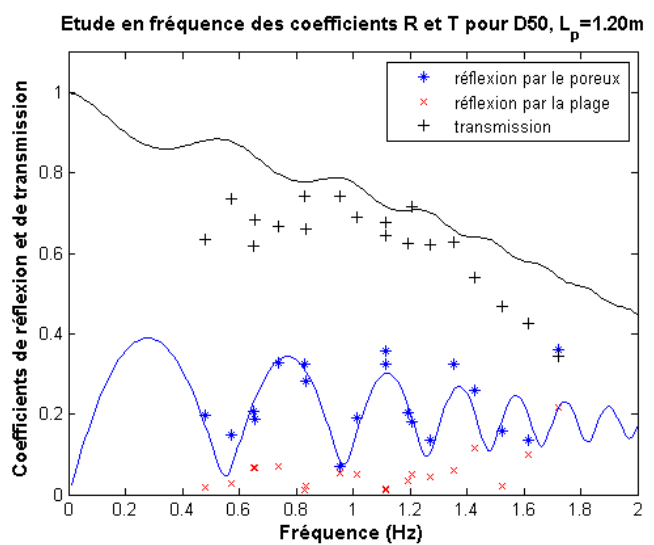

Figure 10. Coefficients de réflexion et de transmission, $L=1.20 \mathrm{~m}, D=0.050 \mathrm{~m}$.

Un bon accord est observé entre la théorie calibrée et les expériences pour le coefficient de réflexion et de ses oscillations relatives au processus d'interférences. En ce qui concerne le coefficient de transmission, le modèle représente de manière satisfaisante la décroissance observée aux plus hautes fréquences, mais ne décrit pas correctement le coefficient de transmission quasi-constant mesuré pour les fréquences inférieures à $1 \mathrm{~Hz}$. Cette différence peut être expliquée par des contraintes techniques: notre générateur de houle ne permet pas un contrôle parfait de l'amplitude des vagues, en particulier pour les basses fréquences. L'amplitude de l'onde mesurée pour les cas évoqués ci-dessus varie de 0.015 à $0.03 \mathrm{~m}$. Des cas tests supplémentaires ont donc été effectués pour mieux appréhender les effets des amplitudes à fréquence donnée, cellesci se situant dans la gamme $0.015-0.055 \mathrm{~m}$, pour une fréquence $f=0.8 \mathrm{~Hz}$, pour le diamètre du cylindre intermédiaire et une structure poreuse de longueur $2.40 \mathrm{~m}$.

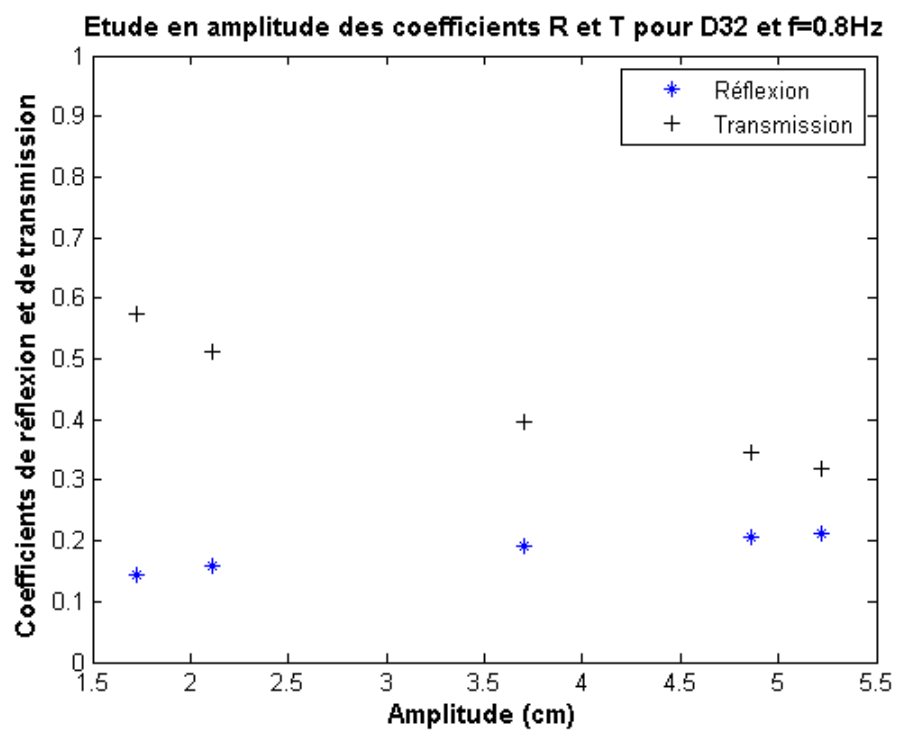

Figure 11. Etude en amplitude des coefficients de réflexion et de transmission. 
Les résultats de la figure 11 montrent que l'amplitude de la houle a un impact beaucoup plus important sur la transmission que sur la réflexion. La tendance principale est une nette diminution du coefficient de transmission avec une amplitude des vagues croissante. Pour les amplitudes les plus faibles, le coefficient de transmission est de l'ordre de 0.5 à 0.6 ce qui est conforme à la valeur pratiquement constante observé lors de l'étude de la fréquence dans la plage des basses fréquences pour la même structure poreuse (voir figure 7). Ainsi, il apparaît plutôt probable qu'au moins une partie de l'écart entre la théorie et le coefficient de transmission expérimental à basse fréquence peut être attribué à l'absence de contrôles sur les conditions expérimentales. Des travaux techniques et expérimentaux supplémentaires seront menés pour examiner ces questions.

\section{Conclusions et perspectives}

La présente étude visait à analyser expérimentalement l'influence de la surface spécifique sur les flux stationnaires et la dynamique des vagues dans un milieu poreux constitué de cylindres verticaux. Les expériences pour les écoulements stationnaires ont d'abord montré l'importance des effets inertiels, qui sont bien représentés en incluant un terme quadratique dans la loi de Darcy généralisée (éq. 3). La principale conclusion de notre étude est la mise en évidence du rôle joué par la surface spécifique tant pour un écoulement stationnaire que pour une houle régulière. La tendance principale est que plus la surface spécifique est importante, plus la dissipation d'énergie est forte. Le modèle de vague proposé à travers la structure poreuse souligne l'importance de deux paramètres clés : la masse ajoutée et le coefficient d'atténuation qui ont une influence significative sur les processus d'interférence (réflexion et transmission) et la dissipation d'onde, respectivement. Une fois ajusté aux données expérimentales, un accord global satisfaisant est trouvé entre le modèle théorique et les mesures. Deux régimes sont cependant observés dans l'évolution du coefficient de transmission à travers la gamme de fréquences. À haute fréquence, la transmission décroît de façon monotone avec l'augmentation de la fréquence, qui est en bon accord avec les prédictions du modèle, tandis que pour les plus longues vagues une valeur presque constante du coefficient de transmission est observée. Cette dernière tendance est probablement liée aux variations de l'amplitude des ondes et des travaux supplémentaires doivent être engagés pour répondre à ces questions.

D’autres améliorations du modèle concernent principalement la mise en œuvre d'un nouveau terme d'atténuation basé sur la dissipation d'énergie par la contrainte de trainée plutôt que le terme courant de décroissance exponentielle. La diminution de l'amplitude de l'onde sera de la forme $1 /(1+B x)$, la constante $B$ étant indépendante de la localisation dans le milieu poreux. Une autre perspective intéressante du présent travail de recherche est l'analyse complète des lois d'échelle de similitude. Par exemple, l'étude 
de modèles réduits poreux pourrait impliquer une variation de surface spécifique avec un changement de régime, même si la porosité est constante.

\section{Remerciements}

Les auteurs aimeraient remercier la région "Provence Alpes Côtes-d’Azur" et la société "Acri-In" pour leur support financier. Un remerciement spécial destiné à Aimed Ajroud pour son aide dans la conception des structures poreuses.

\section{Références}

CALHOUN R.J. (1971). Field study of wave transmission through a rubble-mound breakwater. United States Naval Postgraduate School.

DALRYMPLE R. (1991). Reflection and transmission from porous structures under oblique wave attack. Journal of Fluid Mechanics, Vol. 224, pp 625-644. http://dx.doi.org/10.1017/S0022112091001908

GU Z., WANG H. (1991). Gravity waves over porous bottoms. Coastal Engineering, Vol. 15(5), pp 497-524. http://dx.doi.org/10.1016/0378-3839(91)90025-C

GUYON E., HULIN J.P., PETIT L. (1991). Ecoulements dans les milieux poreux. In Hydrodynamique Physique, Collection Savoirs actuels, interédition/édition du CNRS (pp. 375-384).

MADSEN P.A. (1983). Wave reflection from a vertical permeable wave absorber. Coastal Engineering, Vol. 7, pp 381-396. http://dx.doi.org/10.1016/0378-3839(83)90005-4

MANSARD E., FUNKE E. (1980). The measurement of incident and reflected spectra using a least squares method. Coastal Engineering Proceedings, Vol. 1(17), pp 154-172. MOLIN B. (2011). Hydrodynamic modeling of perforated structures. Applied Ocean Research, Vol. 33(1), pp 1-11. http://dx.doi.org/10.1016/j.apor.2010.11.003

MUNSON B.R., YOUNG D.F., OKIISHI T.H. (1990). Fundamentals of Fluid Mechanics. (2nd Editio., p. 893). New York: John Wiley \& Sons, Inc.

REY V. (1995). A note on the scattering of obliquely incident surface gravity waves by cylindrical obstacles in waters of finite depth. European Journal of Mechanics. B, Fluids, Vol. 14(2), pp 207-216.

REY V., BELZONS M., GUAZZELLI E. (1992). Propagation of surface gravity waves over a rectangular submerged bar. Journal of Fluid Mechanics, Vol. 236, pp 453-479. http://dx.doi.org/10.1017/S0022112092001186

SOLLITT C., CROSS R. (1972). Wave transmission through permeable breakwaters. Coastal Engineering Proceedings, Vol. 1(13), pp 1827-1846.

YU X., CHWANG A. (1994). Wave motion through porous structures. Journal of Engineering Mechanics, Vol. 120(5), pp 989-1008. http://dx.doi.org/10.1061/(ASCE)07339399(1994)120:5(989) 